\title{
Robust trajectory tracking with non-singular terminal sliding mode control of a mobile manipulator
}

\author{
Shankar J. Gambhire ${ }^{1,2}$, Dondapati Ravi Kishore ${ }^{3}$, Pandurang S. Londhe ${ }^{4}$, Sushant N. Pawar ${ }^{5}$ \\ ${ }^{1}$ Research Scholar at Koneru Lakshmaiah Education Foundation, Vaddeswaram, India \\ ${ }^{2}$ Maharashtra Academy of Naval Education and Training, MIT-ADT University, Pune (MH), India \\ ${ }^{3}$ Godavari institute of Engineering and Technology, Rajahmundry, Andhra Pradesh, India \\ ${ }^{4}$ Instrumentation Engineering, Government College of Engineering, Chandrapur, India \\ ${ }^{5}$ Department of Instrumentation, Ramrao Adik Institute of Technology, Nerul, Navi Mumbai, India
}

\section{Article Info}

Article history:

Received Aug 11, 2021

Revised Nov 12, 2021

Accepted Dec 13, 2021

\section{Keywords:}

Mobile manipulator

Non-singularity

Robust control

Terminal-sliding mode

control

\begin{abstract}
This effort addresses and uses a robust-trajectory-tracking control system for kinematically redundant location tracking of a mobile manipulator (mobman) in its task space in the presence of parameter uncertainties and external disturbances. It consists of a serial manipulator with four degrees of freedom (DOF) fixed on three DOF mobile bases. A robust tracking control is achieved using the configuration of a non-singular terminal sliding mode (NSTSM) control for the entire non-linear model of a mobman. Because of the non-linear added term in the linear sliding surface, which reflects in the non-linear sliding mode known as terminal sliding mode (TSM), this control preparation satisfies systems states finite time convergence. Similarly, conventional terminal sliding mode control (CTSMC) entail singularity trouble, which is tackled by the control scheme. (Computer based) simulations are used to demonstrate the convenience, feasibility, and robustness of given techniques.
\end{abstract}

This is an open access article under the CC BY-SA license.

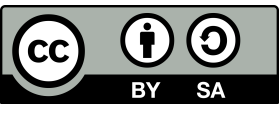

\section{Corresponding Author:}

Shankar J. Gambhire

Research Scholar at Koneru Lakshmaiah Education Foundation

Vaddeswaram, Guntur, AP 522502, India

Email: sjgambhire@gmail.com

\section{INTRODUCTION}

To optimize the robot's reliable and fast-tracking execution, robust quick tolerant-adaptive-control has been planned. It brings a maximum degree of strength, a quick transitory response, and a finite time convergence method [1]. For a given tracking control scheme, a back stepping control tactic is settled to elude singularity-point matter [2]. Trendy master-slave configuration for a group of mobile robots, path planning based on artificial potential field (APF) united with tracking control mechanism based on FAGNTSMC here [3] the authors have submitted. The authors have suggested an adaptive-fast and stable control way to control robotic manipulator with $\mathrm{n}$-degrees of freedom $\mathrm{n}$-(DOF) [4]. In presence of uncertainties, the non-singular terminal sliding mode control (TSMC) is proposed plus applied to a non-linear four-linked selective compliant assembly robot arm (SCARA) robot-manipulator [5]. Mobile-manipulators have been employed effectively to perform not only effective but also interactive practical assignments like drilling operations and laser cutting, choose an object relocation plus complex surface painting or arc welding, peg-hole addition, and over a long time [6]. Collision avoidance compliance and matter of singularity is defined using an external forfeit utility tactic, domino effect in bounded plus continuous control of mobman even near disturbance limits. Using random profile technique, control the movement of the mobile-manipulator so as to complete a task of pick up. 
Which is caused by the mobman's high mobility plus huge workspace area as compared to a fixed base manipulator. By operating in their task space a mobile-manipulator, they can achieve performance stability, high accuracy, and communicative actions. The core impartial of initiate a control is to transfer mobman starting its initial arbitrary place towards aimed position of end effector stated plus great accurateness in chore province coordinates [7]. So far, progress of control algorithms for good tracking enactment is thought-provoking chore on account of dynamic-model of mobman with indeterminate state. Essentially consisting state disparity restraints enforced on mobman crusade and basic uncertain self-motivated equation [8], [9]. Physical uncertainty started by anonymous payload captured by end effector in spite of the fact that the state inequality confinements began in workspace because of the nearness of anonymous issues. The deliberate parametric uncertainties lie in selfmotivated conditions and kinematic of mobile-manipulator. Besides, the mobile controller possesses mobile platforms and strong-coupled elements of the controller. So, the plan of the control system turns an inquisitive subject of current investigation for such sorts of uncertain system. For the moment, researchers have turn up for mobile-manipulators tracking control with control procedures as specified in the literature. Including robust adaptive fault tolerant control, chore-null space decoupling control [10], non-linear closed loop control [11], computed torque control (CTC) [12], neural network (NN) [13]-[15], as a result of complex computation low response time of fuzzy logic control, fuzzy plus NN [16], [17], tracking control that is adaptable and robust, and using a variety of sliding-mode controls [18]. The above study works proposed a self-motivated control law that combines many control procedures such as back stepping, sliding mode, neural-network plus fuzzy logic, making controller design extra laborious, and expensive computationally. Additionally, controllers beginning [19]. Certain controllers in addition to pseudo-inverse [20] require Jacobian-matrix inverse each, within a singular region it may domino effect in mathematical variability. Via Jacobian pseudo inverse general-algorithm specified trendy autogenously space pattern matter of inverse-kinematic can be solved [21]. In the middle of above-mentioned control approaches, sliding mode control (SMC) comprise of terminal sliding mode (TSM), and conventional sliding-mode (CSM). In addition to CSM, TSM is finite time stable plus asymptotically stable [22]. Continuous SMC might ensure asymptotic stability mode, implying that the system states will converge to the equilibrium point over a finite settling time. TSM is a non-linear, also planned as per concept of terminal attractor in order to endorse finite time convergence [23], [24]. TSM, compared with traditional SMC, provides quick besides finite time convergence, making it ideal for high-precision control. Singularity is an issue with TSM controller design procedures. A major reading has been done towards ignoring trouble of singularity via non singular terminal sliding mode power control (NTSMC) [25]. SMC is designed in both stages: the first is to elect a sliding-mode surface, and next to design a SMC to ensure sliding mode presence and drive mechanism to acquire switching manifold in a finite amount of time plus stay there. Now, NTSMC based trajectory tracking control design is presented then used for a mobman complete model (non-linear). This dynamical model of a mobile-manipulator incorporates outer quandaries such as parameter uncertainties, noises, and the sensor used for measurement. The foremost contributions of article are stated is being as: a robust trajectory tracking control is designed for highly nonlinear with 7-dof mobman, robust control is achieved by designing non-singular TSMC under uncertain conditions, property of finite time convergence is demonstrated using Lyapunov method, simulation (numerical) on an assumed manipulator-system is done to validate usefulness of the proposed controller.

\section{RESEARCH METHOD}

\subsection{Dynamics of the mobile manipulator}

Explaining in this effort, said mobman encompass a mobile platform with 4-wheel mounted using a manipulator. 4 motors in helm drives manipulator sordid self-sufficiently. Figure 1 shows projected mobman design, where, moving frame mobile base $M(x, y, z), T\left(x_{t}, y_{t}, z_{t}\right)$ shows end-effector frame, and $I(0,0,0)$ gives inertial frame (earth-fixed). Table 1 shows the mobman structure specifications. To cultivate dynamicmotion equation mode of Newton-Euler given below can be utilized for mobman:

$$
M(q) \ddot{q}+C(q, \dot{q}) \dot{q}+g(q)=\tau+f_{d i s}
$$

where, joint positions manipulator vector $\zeta=\left[\begin{array}{lll}x & y & \psi\end{array}\right]^{T}$ and $q \in \Re^{7 \times 1}$ is joint position vector mutables

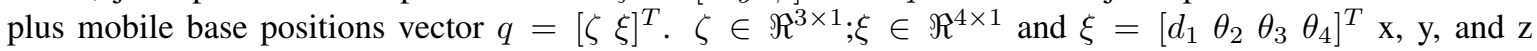
exemplifies translation-positions plus yaw-angular displacement of mobile base. $\theta_{2}, \theta_{3}, \theta_{4}$, and $d_{1}$ analogous 
mobman links angles too joint-displacement of manipulator each. The $f_{(}$dis $)$is vector of lumped disturbance which holds system uncertainties, frictional effects and peripheral difficulties, vector of manipulator moments plus inertial forces: $M(q) \ddot{q}$, centripetal and coriolis vector effects for manipulator: $C(q, \dot{q}) \dot{q}$, vector due to manipulator's gravity-effects: $g(q)$ control-input torques vector: $\tau=\left[\tau_{m} \tau_{b}\right]^{T} \in \Re^{7 \times 1}$, wherever, input torques vector: $\tau_{m} \in \Re^{4 \times 1}$ unstated for serial-manipulator control fixed on mobman plus $\tau_{b} \in \Re^{3 \times 1}$ : input-torque vectors towards control mobile-manipulator base. $\left(f_{d i s}\right)$ : vector of lumped trouble agreed as:

$$
f_{\text {dis }}=f_{\text {idis }}+f_{\text {edis }}
$$

where, $f_{\text {edis }}$ : vector of external-disturbances acting on mobile-manipulator, $f_{\text {idis }}$ : internal disturbances vector despite parametric disturbances besides uncertainties caused as a result of measurement noises and may be given as:

$$
f_{i d i s}=\Delta M(q) \ddot{q}+\Delta(C(q, \dot{q})) \dot{q}+\Delta g(q)+F(q, \dot{q}) \dot{q}+\nu
$$

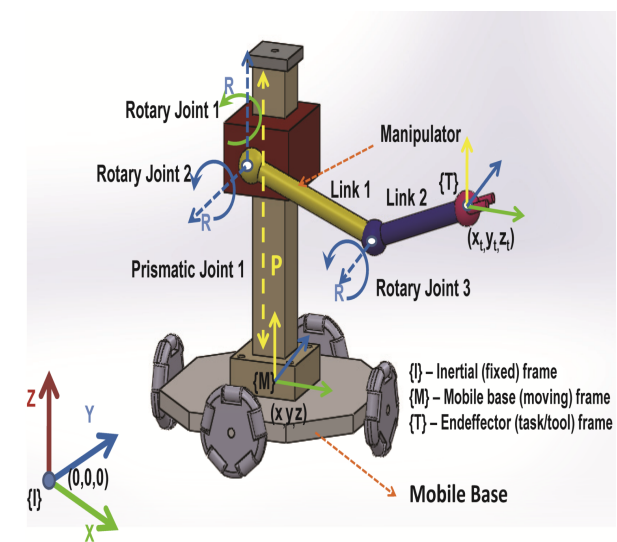

Figure 1. Planned mobman conceptual design

Table 1. Mobman structure specifications

\begin{tabular}{lc}
\hline Speciffication & Value \\
\hline over-all weight of base & $15 \mathrm{~kg}$ \\
over-all height & $300 \mathrm{~mm}$ \\
diameter with circular base & $500 \mathrm{~mm}$ \\
Mobile base Allowable payload & $12 \mathrm{Kg}$ \\
Maximum base velocity & $0.8 \mathrm{~meter} / \mathrm{sec}$ \\
Type & Omni Directional \\
No. of helms & 4 (singly driven) \\
Diameter & $47.5 \mathrm{~mm}$ \\
No. of axix & 4 (PRRR configuration) \\
Height & $1.000 \mathrm{~mm}$ (trendy extended mode) \\
Reach & $500 \mathrm{~mm}$ (trendy extended mode) \\
Work Envelope & $0.625 \mathrm{~m} \mathrm{3}$ \\
Payload & $2 \mathrm{Kg}$ \\
Axis speed & $90 \mathrm{deg} / \mathrm{sec}$ \\
Manipulation link (ML) 1: Length & $300 \mathrm{~mm}$ \\
ML 2: length & $200 \mathrm{~mm}$ \\
Maximum Limit of Prismatic Joints & $500 \mathrm{~mm}$ \\
ML: 1 mass & $5 \mathrm{~kg}$ \\
ML: 2 mass & $3 \mathrm{~kg}$ \\
Actuators & BLDC motors (24 VDC) with $75 \mathrm{~W}$ drive train power limit \\
\hline
\end{tabular}

where $\Delta(M(q)) \ddot{q}, \Delta(C(q, \dot{q})) \dot{q}$, and $\Delta g(q)$ remain signal-uncertainties in $M(q) \ddot{q}, C(q, \dot{q}) \dot{q}$, and $g(q)$ each one. $F(q, \dot{q}) \dot{q}$ vector represents frictional effects (static, coulomb, and viscous) due to joint of manipulator. Vector" $\nu$ " appears inside disturbances presented by position velocity estimation devices. For framework flow, 
earth-fixed vector representation believed through properties of a mobile-controller system. Specified below are finite dimensional.

Property 1. Positive symmetric and definite (PDS) is inertia matrix $M(q)$.

$$
M(q)=M^{T}(q)>0, \forall q \in \Re
$$

Property 2. In addition to $m_{m}$ non-negative constants take place $m_{M}$ i.e. $m_{m} \leqslant\|M(q)\| \leqslant m_{M}$. The matrix $M(q)-2 C(q, \dot{q})$ is a skew symmetric matrix i.e.

$$
s^{T}[M(q)-2 C(q, \dot{q})] s=0, \forall s \in \Re, q \in \Re
$$

\subsection{Control system design}

By way of forcing the states $q$ for tracking the desired-position $q_{d}$ is the objective of this control. Definition of tracking error can be given as,

$$
e=q_{d}-q
$$

where, desired trajectory is $q_{d}$. As per (1) designation of a terminal siding surface (non-singular).

$$
s(t)=e(t)+\left(\frac{1}{\beta}\right) \dot{e}(t)^{\frac{p}{q}}=0 .
$$

Where $\beta=\operatorname{diag}\left[\beta_{1}, \cdots, \beta_{i}, \cdots, \beta_{n}\right]$ in which a positive constant is $\beta_{i}, p$ and $q$ are non-negative odd integers , which satisfy the situation as given as:

$$
p>q .
$$

Condition for sufficiency to demonstrate existence of TSM equally:

$$
\frac{1}{2} \frac{d}{d t} s_{i}(t)^{2}<-\zeta_{i}\left|s_{i}(t)\right|
$$

where positive constant is $\zeta_{i}(i=1, \cdots, n)$. TSM is mentioned in (1): $\boldsymbol{s}_{n}(t)=0$ its dynamics utilising nonlinear differential equation gained:

$$
-\dot{e}(t)^{\frac{p}{q}}=e(t) \beta
$$

Infinite period of time with $\mathrm{e}(\mathrm{t})$ approach terminal-sliding surface $t_{r_{i}}(i=1, \cdots, n)$, as $s(t) \neq 0$, which fulfills:

$$
t_{r_{i}} \leqslant \frac{\left|s_{i}(0)\right|}{\zeta_{i}}
$$

Mobman structure is stable when NTSMC control law chosen in place of supposing NTSMC, sufficient condition given in (7) and (9):

$$
\tau=\left(\ddot{q}_{d}+\beta \frac{q}{p} \dot{e}(t)^{2-\frac{p}{q}}+\zeta \operatorname{sgn}(s)\right) M(q)+C(q, \dot{q}) \dot{q}+g(q)
$$

$\operatorname{sgn}(s)$ discontinuous function (Df) as per above equation chattering of control-input will be provided. Chattering is a detrimental phenomenon as it leads to decrease control accuracy, moving mechanical parts high wear too power circuits with high heat losses. Exploitation of digital controllers with finite sampling rate is chattering, also called 'discretization- chatter'. Ideally best sliding mode hint at infinite switching frequency. Subsequently within a sampling interval control is constant, switching frequency cannot exceed that of sampling, which lead to chattering as well increases the energy consumption of the controller. This Df $\operatorname{sgn}(s)$ replaced through continuous $\operatorname{sat}(s, \Phi)$ called saturation-function, to compensate undesirable control chattering, can be defined: where, boundary layer thickness is $\Phi_{i}$.

$$
\{\operatorname{sat}(s, \Phi)\}_{i}= \begin{cases}\frac{s_{n_{i}}}{\left|s_{n_{i}}\right|} & \text { if } s_{n_{i}}>\Phi_{i} \\ \frac{s_{n_{i}}}{\Phi_{i}} & \text { if } s_{n_{i}} \leq \Phi_{i}\end{cases}
$$




\subsection{Stability analysis}

Now, 'Lyapunov-stability analysis' way is useful to reveal convergence property of offered NTSMC controller. Assumptions made to elucidate stability-analysis of given NTSMC controller, could be as shown in. assumption 1. Term well-known as $f_{\text {dis }}$ lumped disturbance is bounded, be made up of constant $0 \leq\left|f_{\text {dis }}\right| \leq F_{\max }$.

assumption 2. $\dot{q}$ and $q$ states are available to measure the quantity which is to be measured.

assumption 3. $q_{d}$ required trajectory is smooth along with double-differential i.e. $q_{d}, \dot{q}_{d}, \ddot{q}_{d}$ too reachable by means of well-identified boundaries.

\subsubsection{Theorem}

Take up both properties with mobile-manipulator dynamics (1). Vector of controlinput is taken as defined (12). Error vector monitoring output touches to zero in an in-finite amount of-time with respect to $s$.

\subsubsection{Proof}

Take up Lyapunov-candidate function recognised for instance,

$$
V=\frac{1}{2} M s^{T} s
$$

Taking derivative of $V$ from (13), it becomes,

$$
\dot{V}=\frac{1}{2}\left\{\dot{s}^{T} M s+s^{T} \dot{M} s+s^{T} M \dot{s}\right\}
$$

Since $s^{T} M \dot{s}=\dot{s}^{T} M s, \dot{M}=2 C(q, \dot{q})$ (property 2) can be solved as,

$$
\begin{gathered}
\dot{V}=s^{T}\{M \dot{s}+C(q, \dot{q}) s\} \\
\dot{V}=s^{T}\left\{M\left(\dot{e}(t)+\frac{1}{\beta} \frac{p}{q} \operatorname{dia}\left(\dot{e}(t)^{\frac{p}{q}-1}\right) \ddot{e}(t)\right)+C(q, \dot{q}) s\right\} \\
\ddot{e}(t)=\ddot{\eta}_{d}-\ddot{\eta}
\end{gathered}
$$

As mentioned in (1) and (12), it gives,

$$
\ddot{e}(t)=-\frac{q}{p} \beta\left(\operatorname{dia}\left(\dot{e}(t)^{2-\frac{p}{q}}\right)\right)-\zeta \operatorname{sgn}(s)-M^{-1} f_{d i s}
$$

Putting value of $\ddot{e}(t)$ to 16 through $[18$ it becomes,

$$
\dot{V} \leqslant s^{T}\left\{\frac{1}{\beta} \frac{p}{q} \operatorname{dia}\left(\dot{e}(t)^{\frac{p}{q}-1}\right)\left[-M \zeta \operatorname{sgn}(s)-f_{d i s}\right]\right\}+s^{T} C(q, \dot{q})^{T} s
$$

$1<\frac{p}{q}<2$ as $p$ and $q$ are positive odd integers, there is $\dot{e}_{i}(t)^{\frac{p}{q}-1}>0$ so, negative definite would be Lyapunovfunction (V) time-derivative if and $V$ fulfils differential inequality as per (20) as shown in (21),

$$
\begin{gathered}
\left|\zeta_{i}\right|>\left|\tau_{d i s_{i}}\right| \\
\dot{V}(t) \leq V(t)^{\alpha}(-\gamma)
\end{gathered}
$$

It consists, $\alpha \in(0,1)$ plus $\gamma>0$. In (22), the parameters $\alpha$ and $\gamma$ are defined such that to have (22) as negative definite and hence stable. Here, the value of $\gamma$ has to be positive constant to ensure negative definiteness of (22) and hence considered as $\gamma_{i} 0$. To have convergence of $\mathrm{V}$ with respect time towards zero, the value of parameter $\alpha$ has to be selected between 0 and 1. Hereafter, as per Lyapunov-stability standards converges to zero infinite time a non-linear plus non-singular sliding surface given in (7). 


\section{RESULTS AND DISCUSSION}

\subsection{Description of planned structure}

In-depth computer-based simulations were conducted to demonstrate potential of control strategy on offered robotic framework. Given controller framework comprise of 4 DOF (PRRR) mounted on 3 DOF mobile-base of serial controller. Test-case is picked so that manipulator begins on starting point, reaching-back to the initial point afterwards roving a given multipart set way. During activity, the controller selected uncertain mass body and positioned it in a specific location (i.e. $2 \mathrm{KG}$ pay-load engaged into thought for recreation), transmit mass of load (un-identified) along the aforementioned foreordained way in addition put the question at focused on point of the area.R) mounted on 3 DOF mobile-base of serial controller.

\subsection{Performance analysis}

This sub-division, Figures 2-5 denotes computer-based simulation outputs for chore space trajectory revealed overhead. Deviation in payload is from $0 \mathrm{~kg}$ to $2.5 \mathrm{~kg}$ (no load to payload full). In addition, structure uncertainty differs $(-40 \%$ to $40 \%)$, controller robustness has been specialized to get a suitable considerate of scheme robustness, over and above scheme uncertainty vector of external-disturbance is included, and is presented is being as,

$$
\delta_{d i s}=\left[\begin{array}{l}
5 \cos \left(w_{d} t\right) \\
5 \sin \left(w_{d} t\right) \\
5 \cos \left(w_{d} t\right)
\end{array}\right] \text { in } \mathrm{N}
$$

Where, external disturbance frequency is $w_{d}$, brings out simulations to find performance of controller in case of speedy disturbance change and changes (0-2 rad/s. Comparative study has been supported to appear the legitimacy of the proposed strategy, with renowned controllers alike linear proportional+integral+derivative (PID) CTC. For mentioned controllers control laws can be expressed as:

$$
\begin{aligned}
& \eta=K_{P} \tilde{q}+K_{I} \int \tilde{q} d t+K_{D} \frac{d \tilde{q}}{d t}: \text { PID } \\
& \eta=\hat{M}(q)\left(\ddot{q}_{d}+K_{1} \dot{\tilde{q}}+K_{2} \tilde{q}\right)+\hat{C}(q, \dot{q}) \dot{q}+\hat{g}(q): \text { CTC }
\end{aligned}
$$

To achieve a better comparison, the following errors and controller gain matrices are set to be about the same to facilitate altogether controllers agreeable control execution under archetypal situations. (i.e. $f_{\text {dis }}=0$ ). Controller LPID variables: $K_{P}=6.5, K_{I}=3.5, K_{D}=1.25$; for CTC, $K_{1}=4.56, K_{2}=6.32$. In lieu of suggested NTSMC: $q=3, \beta=8, p=5, \zeta=15$, and $\Phi=0.009$. The three-dimensionless view of tracking responses given by all the control methods have been shown in Figure 2. It can be clearly seen that proposed controller shows better tracking performance compared with PID and CTC methods. For complex-chore space position-controllers relational result the following control at the side uncertain condition as appeared in Figure 3. It may say that controller viably takes after desired multipart route also brings out moving object at same time, as per result. The CTC and LPID ways offer significant inaccuracies with trajectory following, inadequately in overseeing external-disturbances plus parameter-uncertainties. Noticing chore-space time histories inaccuracies set by controllers established plus time trajectories of tracking-error norm (Euclidean-norm) are shown in Figure 4 and justifies the pitched scheme performance. Figure 5 shows the control efforts given by proposed control method. A quantitative analysis of the tracking performance of a mobman given by all the controllers in terms of integral of the absolute error (IAE), root mean square of the error (RMS), and integral of the time absolute error (ITAE) is done and given in Table 2 from which, it is confirmed that as compared to other controllers the proposed control scheme gives minimum tracking errors in $\mathrm{x}, \mathrm{y}$, and $\mathrm{z}$ directions.

Table 2. Performance indices of conntrollers

\begin{tabular}{llllllllll}
\hline \multirow{2}{*}{ Control Schemes } & \multicolumn{3}{c}{$\tilde{\mu_{x}}$} & \multicolumn{3}{c}{$\tilde{\mu_{y}}$} & \multicolumn{3}{c}{} \\
\cline { 2 - 10 } & RMS & IAE & ITAE & RMS & IAE & ITAE & RMS & IAE & ITAE \\
\hline LPID & 0.0675 & 55.5897 & 24839 & 0.0574 & 51.6067 & 26985 & 0.0586 & 51.9647 & 24824 \\
CTC & 0.0286 & 21.6217 & 9371.9 & 0.022 & 19.9124 & 10362 & 0.0245 & 20.5275 & 9382.7 \\
NTSMC & 0.0019 & 0.3511 & 78.8374 & 0.00052 & 0.1646 & 72.6711 & 0.0029 & 0.6718 & 313.3997 \\
\hline
\end{tabular}




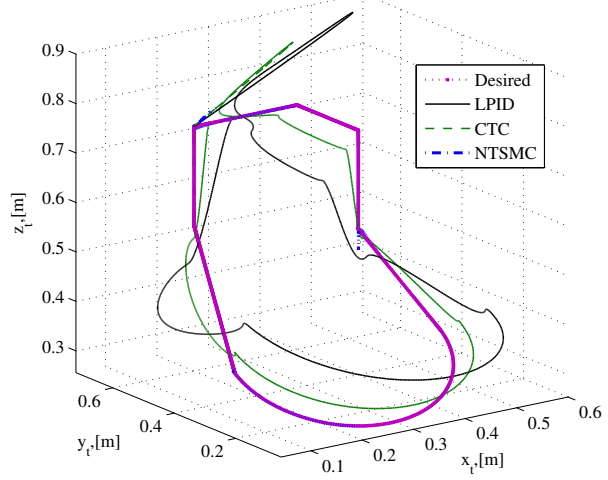

Figure 2. Trajectory tracking-control (TTC) with inde-finite condition (3D view)

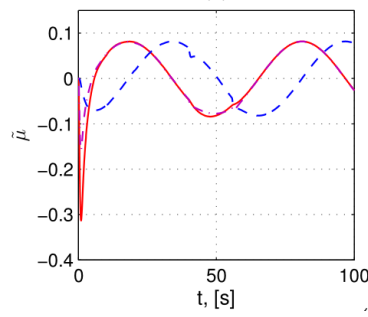

(a)

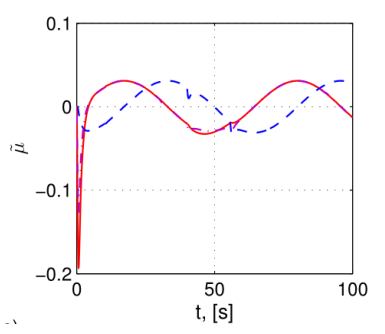

(b)

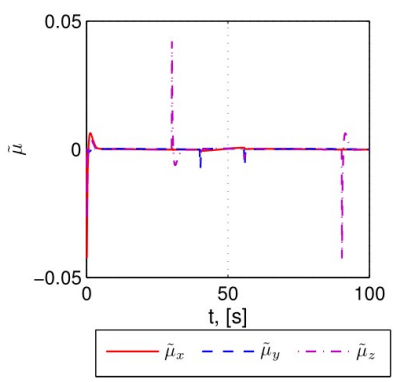

(c)

Figure 3. The time history for tracking task space deviations by (a) LPID, (b) CTC, and (c) NTSMC method

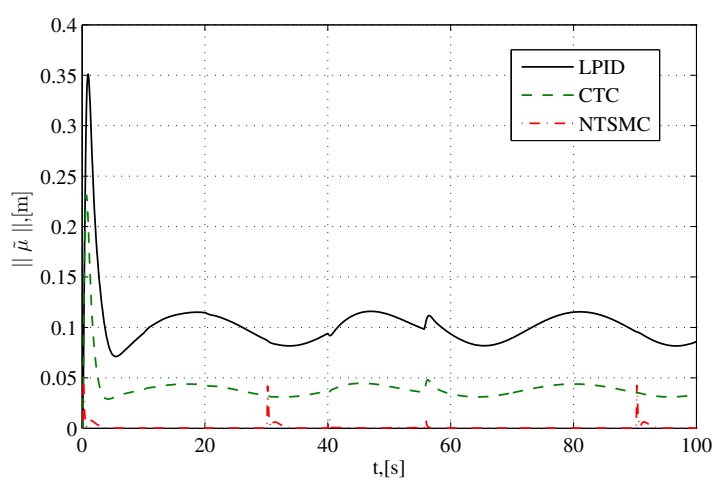

Figure 4. Deviancies in norm variations of the tracking location all through multipart chore space trajectory-tracking control thru indeterminate condition 


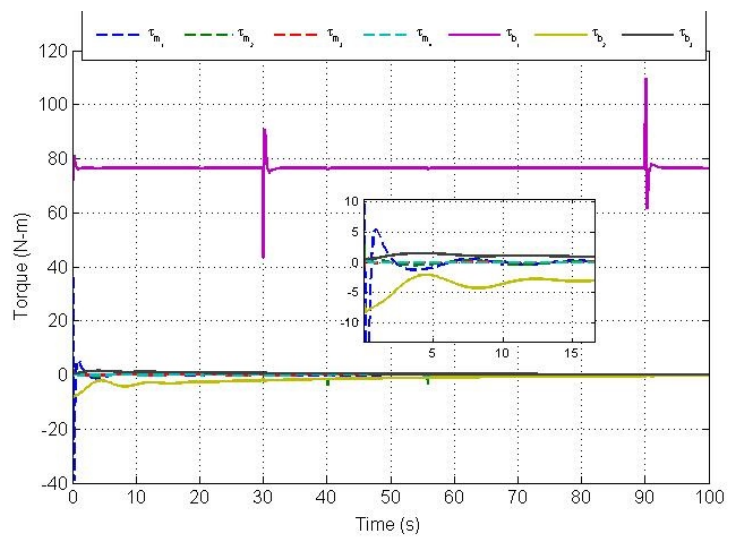

Figure 5. Control efforts during tracking control by proposed method

\section{CONCLUSION}

For combined prearranged chore-space motion control of 7-DOF manipulator mode (mobile), a nonsingular non-linear robust TSM control algorithm is presented as well as useful. The utility of submitted way has been verified by matching results to that of existing controllers. The proposed performance better in form of tracking, finite time error convergence plus robustness, according to simulation results.

\section{REFERENCES}

[1] M. Van, M. Mavrovouniotis, and S. S. Ge, "An adaptive backstepping nonsingular fast terminal sliding mode control for robust fault tolerant control of robot manipulators," in IEEE Transactions on Systems, Man, and Cybernetics: Systems, vol. 49, no. 7, pp. 1448-1458, July 2019, doi: 10.1109/TSMC.2017.2782246.

[2] S. Hou and J. Fei, "Adaptive fuzzy-neural control of active power filter using nonsingular terminal sliding mode controller," IECON 2017-43rd Annual Conference of the IEEE Industrial Electronics Society, 2017, pp. 6504-6509, doi: 10.1109/IECON.2017.8217133.

[3] R. R. Nair, H. Karki, A. Shukla, L. Behera, and M. Jamshidi, "Fault-Tolerant Formation Control of Nonholonomic Robots Using Fast Adaptive Gain Nonsingular Terminal Sliding Mode Control,' in IEEE Systems Journal, vol. 13, no. 1, pp. 1006-1017, March 2019, doi: 10.1109/JSYST.2018.2794418.

[4] A. Rezoug and M. Hamerlain, "Adaptive neural higher order terminal sliding mode controller application to trajectory tracking of robotic manipulator," 2016 8th International Conference on Modelling, Identification and Control (ICMIC), 2016, pp. 320-325, doi: 10.1109/ICMIC.2016.7804130.

[5] Y. Yang, H. Chen, and L. Zhang, "Nonsingular terminal sliding-mode control for nonlinear robot manipulators with uncertain parameters," 2015 IEEE International Conference on Robot. and Biom., 2015, pp. 1227-1232, doi: 10.1109/ROBIO.2015.7418939.

[6] M. Galicki, "Two-stage constrained control of mobile manipulators," Mechanism and Machine Theory, vol. 54, pp. 18-40, Aug. 2012, doi: 10.1016/j.mechmachtheory.2012.04.001.

[7] M. Galicki, "Task space control of mobile manipulators," Robotica, vol. 29, no. 2, pp. 221-232, Mar. 2011, doi: 10.1017/S026357471000007X.

[8] P. Štibinger, et al., "Mobile manipulator for autonomous localization, grasping and precise placement of construction material in a semi-structured environment," in IEEE Robotics and Automation Letters, vol. 6, no. 2, pp. 2595-2602, April 2021, doi: 10.1109/LRA.2021.3061377.

[9] H. Zhang, Q. Sheng, Y. Sun, X. Sheng, Z. Xiong, and X. Zhu, "A novel coordinated motion planner based on capability map for autonomous mobile manipulator," Robotics and Autonomous Systems, vol. 129, p. 103554, July 2020, doi: 10.1016/j.robot.2020.103554.

[10] G. D. White, R. M. Bhatt, and V. N. Krovi, "Dynamic redundancy resolution in a nonholonomic wheeled mobile manipulator," Robotica, vol. 25, no. 2, pp. 147-156, Mar. 2007, doi: 10.1017/S0263574706003328.

[11] S. Mishra, P. S. Londhe, S. Mohan, S. K. Vishvakarma, and B. M. Patre, "Robust task- space motion control of a mobile manipulator using a nonlinear control with an uncertainty estimator," Computers Electrical Engineering, vol. 67, pp. 729-740, Apr. 2018, doi: 10.1016/j.compeleceng.2017.12.018.

[12] G. Perumalsamy, D. Kumar, J. Jose, S. J. Winston, and S. Murugan, "Computed torque control method for two-axis planar serial robotic arm," IETE Journal of Research, pp. 1-9, 2021, doi: 10.1080/03772063.2020.1869595.

[13] L. Cheng, Z. Wang, F. Jiang, and J. Li, ”Adaptive neural network control of nonlinear systems with unknown dynamics," Advances in Space Research, vol. 67, no. 3, pp. 1114-1123, Feb. 2021, doi: 10.1016/j.asr.2020.10.052.

[14] J. Hernandez-Barragan, J. D. Rios, J. Gomez-Avila, N. Arana-Daniel, C. Lopez- Franco, and A. Y. Alanis, ”Adaptive neural pd controllers for mobile manipulator trajectory tracking," PeerJ Computer Science, vol. 7, p. e393, 2021, doi: 10.7717/peerj-cs.393.

[15] A. H. Khan, S. Li, D. Chen, and L. Liao, "Tracking control of redundant mobile manipulator: an rnn based metaheuristic approach," Neurocomputing, vol. 400, pp. 272-284, Aug. 2020, doi: 10.1016/j.neucom.2020.02.109.

[16] Z. Li and W. Chen, "Adaptive neural-fuzzy control of uncertain constrained multiple coordinated nonholonomic mobile manipula- 
tors," Engin. Appli. of Artificial Intelligence, vol. 21, no. 7, pp. 985-1000, 2008, doi: 10.1016/j.engappai.2007.08.007.

[17] Yu-Xiang Wu, Y. Feng, and Yue-Ming Hu, ’Dynamical adaptive sliding mode output tracking control of mobile manipulators," 2005 International Conference on Machine Learning and Cybernetics, 2005, pp. 731-736 Vol. 2, doi: 10.1109/ICMLC.2005.1527040.

[18] D. Qureshi and M. Salim, "A comprehensive survey of sliding mode-based control methodologies for robotic manipulator," SSRN, June 2020.

[19] V. Padois, J-Y Fourquet, and P. Chiron, "Kinematic and dynamic model-based control of wheeled mobile manipulators: a unified framework for reactive approaches," Robotica, vol. 25, no. 2, pp. 157-173, Mar. 2007, doi: 10.1017/S0263574707003360.

[20] Y. Yamamoto and X. Yun, "Coordinating locomotion and manipulation of a mobile manipula- tor," [1992] Proceedings of the 31st IEEE Conference on Decision and Control, 1992, pp. 2643-2648 vol.3, doi: 10.1109/CDC.1992.371337.

[21] K. Tchoń and J. Jakubiak, "Endogenous configuration space approach to mobile manipulators: a derivation and performance assessment of jacobian inverse kinematics algorithms," International Journal of Control, vol. 76, no. 14, pp. 1387-1419, 2003, doi: $10.1080 / 0020717031000149942$.

[22] ST Venkataraman and S Gulati, "Chattering free full-order sliding-mode control," Automatica, vol. 50, no. 4, pp. 1310-1314, Apr. 2014, doi: 10.1016/j.automatica.2014.01.004.

[23] ST Venkataraman and S Gulati, "Control of nonlinear systems using terminal sliding modes," J. Dyn. Sys., Meas., Control., vol. 115, no. 3, pp. 554-560, Sept. 1993, doi: 10.1115/1.2899138.

[24] M. Zhihong, A. P. Paplinski, and H. R. Wu, "A robust MIMO terminal sliding mode control scheme for rigid robotic manipulators," in IEEE Transactions on Automatic Control, vol. 39, no. 12, pp. 2464-2469, Dec. 1994, doi: 10.1109/9.362847.

[25] Y. Feng, X. Yu, and Z. Man, ”Non-singular terminal sliding mode control of rigid manipulators," Automatica, vol. 38, no. 12, pp. 2159-2167, Dec. 2002, doi: 10.1016/S0005-1098(02)00147-4.

\section{BIOGRAPHIES OF AUTHORS}

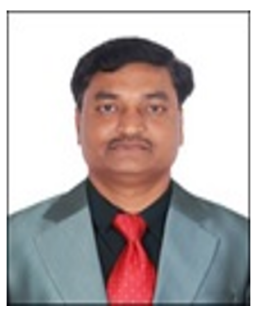

Shankar J. Gambhire (iD) 5 P received B.E. in Instrumentation-Engg. from MBES COE Ambajogai, Dr BAMU, Aurangabaad, India, in 2000 and M.E. in Process-Instrumentation from VIT, Pune, in 2007. Presently, he is research scholar at KLEF, Vaddeswaram, Guntur, AP, and Assistant Professor at MIT ADT University-MANET, Pune, India. He can be contacted at email: sjgambhire@ gmail.com.

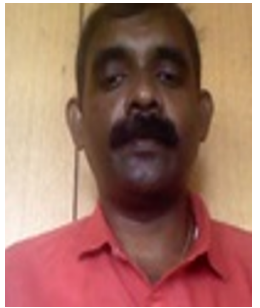

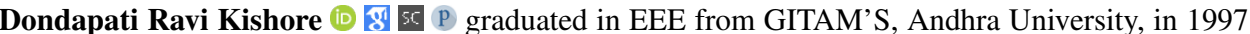
and M.Tech. in Energy-Systems in 2001 plus Ph.D. degree in 2015 from JNTU, Hyderabad, India. He has published 31 papers in international journals and presented 9 in conferences. He is a reviewer of many reputed international journals and submitted 3 research proposal. His areas of interest are Renewable energy, sources power systems and power electronics. Presently, he is Professor and Head of EEE, GIET, NH16, Rajahmundry, AP 533296. India. He can be contacted at email: dravikishore@gmail.com.

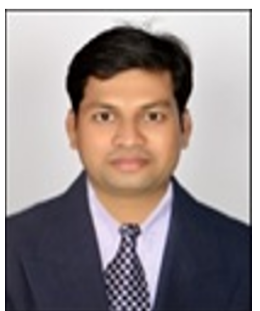

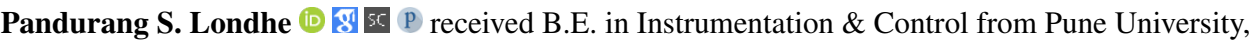
India, (2011), M.Tech. in Instrumentation-Engg. from Marathwada University, Nanded in 2013 and $\mathrm{Ph}$.D. in control of autonomous under-water robots at the SGGS IE\& T, Nanded, Maharashtra, India in 2018. He has 25 plus publication in international journals and national/international conferences. $\mathrm{He}$ is a reviewer of numerous prestigious international journals. His area of interest consists Intelligent-control of under-water robots and control of nuclear-reactor. Currently, he is an Assistant Professor of Instrumentation Engineering at the Government College of Engineering, Chandrapur, India. He can be contacted at email: pandurang197@gmail.com.

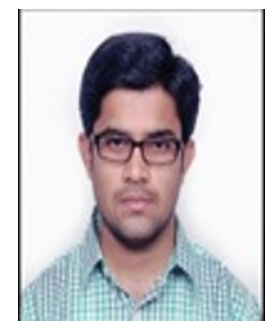

Sushant N. Pawar (D) 80 S 5 has completed his B.E. (Instrumentation \& control) from University of Pune in 2010. He did his M.Tech. in Instrumentation-Engg. from SGGS IE\& T Nanded and Ph.D. in Control System at the SGGS IE\& T, Nanded, Maharashtra, India in Nov,2021. His area of Interest in Process control, Observer based control, Data analytics. He can be contacted at email: sushantnpawar@gmail.com. 